\title{
Hirntumoren - seltene Erkrankungen in der Ära molekular basierter Therapien
}

$\mathrm{T}$

rotz ihrer relativen Seltenheit gehören Hirntumoren aktuell zu den vieldiskutierten und sowohl hinsichtlich des weiteren diagnostischen als auch therapeutischen Procedere kontrovers besprochenen Tumoren. Internationale Statistiken zeigen seit einigen Jahren zudem einen langsamen, aber kontinuierlichen Anstieg in der Inzidenz, vor allem bei älteren Patienten. Ein wichtiger, bereits die niedergelassene Praxis betreffender Bereich in der Diagnostik von Hirntumoren ist die Schnittbildgebung. Hier sollen in den nächsten Jahren durch Implementierung aktueller magnetresonanztomographischer (MRT) Protokolle eine weitere Verbesserung in der Differenzierung nieder- bzw. höhergradiger Gliome sowie eine klarere Abgrenzung zu Metastasen, primären extranodalen Lymphomen des zentralen Nervensystems (PZNSL) und anderen Pathologien erreicht werden. Zudem soll die Wertigkeit der MRT prospektiv zur Verlaufsbeobachtung jenseits der kontrastangehobenen T1-Sequenzen auch für die neuen, antiangiogenen Substanzen untersucht werden. International wird derzeit an einer Revision der Macdonald-Kriterien gearbeitet, die sowohl das Problem der Pseudoprogression als auch die ungewöhnlichen Progressionsmuster nach Therapie mit antiangiogenen Substanzen, wie z.B. Bevacizumab (Avastin ${ }^{\circledR}$ ), Cediranib oder Enzastaurin, berücksichtigt.

In Behandlung und Prophylaxe des häufig zu neurologischen Ausfällen führenden perifokalen Hirnödems kommt mit der Einführung neuer antiangiogener Substanzen nach Jahrzehnten des Stillstands Bewegung. In der Praxis gelingt es aufgrund der durch eine Behandlung mit dem monoklo- nalen Antikörper Bevacizumab erzeugten antiödematösen Effekte häufig, die zuvor notwendige, jedoch nebenwirkungsreiche hochdosierte Steroidtherapie zu beenden und so die steroidbedingte Morbidität und Einschränkung der Lebensqualität zu reduzieren.

Das Thema Methylguanyl-Methyltransferase (MGMT) ist wegen der prognostischen und prädiktiven Aussagekraft, der Kontroverse um die optimale Bestimmung des MGMT-Status und der Pars-pro-toto-Situation bei der Etablierung weiterer prädiktiver und prognostischer Marker wichtig. Nachdem das Dogma, dass eine Bestrahlung eines Hirntumors nur einmal erfolgen kann, gefallen ist, soll jetzt in der European Organization for Research and Treatment of Cancer (EORTC) eine erste Studie zur Redizivstrahlentherapie durchgeführt werden. Aktuelle Studien untersuchen Temozolomid in der Monotherapie bei niedergradigen Gliomen (EORTC 22033/26033), in der Kombination mit Strahlentherapie bei anaplastischen Gliomen (CATNON, EORTC 26052/22054) sowie im Rezidiv, dosisintensiviert nach Versagen einer primären Temozolomidtherapie bei Glioblastomen (DIRECTOR). Weitere wichtige Entwicklungen sind die z.T. nach dem MGMTStatus einschließenden Studien zu antiangiogenen Signalweginhibitoren. Obwohl die erste Phase-III-Studie eines Signalweginhibitors, Enzastaurin, bei Hirntumoren kein positives Resultat erbrachte, erwarten wir nach der Temozolomidära jetzt einen weiteren Fortschritt in der Behandlung von Hirntumoren durch die Implementierung von Signalweginhibitoren in der Primär- oder Rezidivtherapie. Die diesbezüglich aktuell aktiven Studien sind in der Primärsituation bei Patienten mit Glioblastomen mit nichtmethyliertem MGMT-Promotor die deutsche S039-Studie mit Enzastaurin sowie die EORTC-26082/22081Studie mit dem Inhibitor des ,mammalian target of rapamycin“ (mTOR), Temsirolimus $\left(\right.$ Torisel $^{\mathbb{R}}{ }^{\text {}}$. Für Patienten mit Glioblastomen mit methyliertem MGMT-Promotor rekrutiert zurzeit eine gemeinsam von der EORTC und der Firma Merck durchgeführte internationale Phase-III-Studie (CENTRIC) mit dem $\alpha_{v} \beta_{3}$-Hemmstoff Cilengitide.

Bei den PZNSL sind es nicht die Signalweginhibitoren, welche die aktuelle Entwicklung und auch die künftigen klinisch-wissenschaftlichen Konzepte bestimmen, sondern die Fragen, ob und in welcher Form die Radiotherapie zur Primärtherapie gehört, wie ,älter“ bzw. ,jünger“ definiert sind, ob PZNSL wirklich auf das ZNS beschränkt sind, wie der Standardarm für eine zukünftige Primärtherapiestudie aussehen sollte und ob sich molekulare Parameter identifizieren lassen, welche ein Ansprechen auf die Therapie vorhersagbar machen.

Nach Jahren effektiver Grundlagenforschung ohne wesentliche Übertragung in die Klinik stehen wir bei den Hirntumoren am Anfang einer neuen Ära. Aufgrund ihrer immunologischen, zellbiologischen und klinischen Spezifika sind Hirntumoren nur noch selten letzte Spielwiese für wenig vielversprechende Therapien, sondern vielmehr paradigmatische Erkrankungen als Schrittmacher in der Entwicklung zielgerichteter Therapien, meint

Wolfgang Wick 